\title{
HG-MN STARS VERSUS NORMAL STARS
}

\author{
M.M. DWORETSKY \\ Department of Physics and Astronomy, University College London, \\ Gower Street, London WC1E 6BT, UK
}

ABSTRACT The literature concerning spectroscopic studies, abundances, photometry and systematics of $\mathrm{Hg}-\mathrm{Mn}$ stars is reviewed since the last international meeting on chemically peculiar stars in the Crimea in 1985, and is intended to be complete to the end of 1991.

\section{INTRODUCTION}

Seven years ago (Dworetsky, 1986), I provided for IAU Colloquium No. 90 in the Crimea a review of $\mathrm{Hg}-\mathrm{Mn}$ and normal stars, and I shall do the same thing today. Loosely speaking, this means stars with $9,500 \mathrm{~K} \leq \mathrm{T}_{\text {eff }} \leq 16,000 \mathrm{~K}$, i.e. A0 to B5 stars, but because the boundaries of the Hg-Mn phenomena are slightly vague and there are related objects to be discussed, I will allow myself the liberty of relaxing the limits slightly in order to discuss certain objects or phenomena. At this conference, several invited papers overlap this review. I have found it impossible to ignore abundances or recent results from the ultraviolet. I will first discuss normal stars, then the $\mathrm{Hg}$-Mn stars, with emphasis on specific elements and on the more general problem of their observed properties and systematics.

There are several review papers in the literature which cover some of the same ground. Particularly useful are those of Faraggiana and Gerbaldi (1990), and the review by Takada-Hidai (1991). I would also recommend that those interested in abundance analysis problems, which includes many at this colloquium, take a close look at the publication Elemental Abundance Analyses (edited by Adelman and Lanz, 1988).

\section{NORMAL STARS}

We have come to regard normal stars as those which we observe in order to provide a baseline comparison with peculiar stars, so that we can ensure that our abundance analyses and any errors contained therein are differential with respect to stars of similar temperature. However, our overall concept of a normal star as one which has a solar composition is increasingly difficult to maintain. The spread of abundances observed in what we have previously regarded as normal stars is surprisingly large and some elements, such as carbon, have shown themselves to have a few surprises.

All our abundance determinations are ultimately compared to those in Solar System material. Grevesse (1991) showed that in general there was very good 
agreement between results derived from carbonaceous chondrites and from solar spectroscopy. The one exception proved to be Fe (see also Booth, 1989). In the compilation by Anders and Grevesse (1989), which we can take as our solar standard, $\log A_{F e}=7.51$ from meteorites, but the spectroscopic results gave $\log \mathrm{A}_{\mathrm{Fe}}=7.67$. However, recent authors (Biémont et al., 1991; Holweger et al., 1991; Hannaford et al., 1992) now agree that the abundance of Fe in the Sun is somewhat lower than the previously determined value and their new analyses with recently derived $\mathrm{FeI}$ and Fell oscillator strengths show that $\log \mathrm{A}_{\mathrm{Fe}}=7.48$, essentially in perfect agreement with the meteoritic value. This means that we have to reinterpret, in some cases, statements that $\mathrm{Fe}$ is either rich or poor in certain stars. (Note that in this review, abundances will usually be quoted on the scale where $\log N_{H}=12.00$. In a few cases an explicit abundance ratio will be given, or the square bracket notation will be used to express a logarithmic ratio with respect to the solar abundance.)

\section{Published Analyses of Normal Stars}

Adelman and Fuhr (1985) revised previous analyses of normal early A and B stars in the light of improved and critically evaluated oscillator strengths. This rediscussion produced good agreement between abundances from FeI and FeII lines, although Fel tended to give slightly higher abundances by $\sim 0.1$ dex in some cases. The value of $\log A_{F e}$ ranged from 7.24 to 7.64 , which neatly brackets the "new" solar value of 7.50. Further LTE analyses of superficiallynormal and normal stars by Adelman are of $\theta$ Leo, $\tau$ Her, o Peg, 14 Cyg and 5 Aqr with co-added DAO spectra $(1986,1988 \mathrm{a})$ and a second revision of previous work (1988b). Recently, Adelman (1991) has re-investigated four late B normal stars using Reticon spectra obtained at DAO. In general, for these and other stars discussed, the Fe abundance for normal stars ranges from solar to 3 times less than solar; the LTE analyses give close agreement between FeI and FeII, and for the stars with $\mathrm{T}_{\mathrm{eff}}<10,000 \mathrm{~K}$ the derived value of microturbulence $\left(\xi_{\mathrm{t}}\right)$ was essentially $0 \mathrm{~km} / \mathrm{s}$. Some early A stars studied (e.g. o Peg, $\theta$ Leo) have the subtle abundance signatures of hot Am stars: low Sc abundances, with moderately enhanced or normal Fe but high $\mathrm{Sr}, \mathrm{Y}, \mathrm{Zr}$ and $\mathrm{Ba}$. Savanov (1985b) found similar Am signatures in his analyses of $\eta$ Oph, $\theta$ Leo and $\varepsilon$ Ser, and for 43 Peg (1985a). Ptitsyn and Ryabchikova (1986) analysed the B star HR 8226 (HD 204754) and found that its composition is very similar to $v$ Cap, a late $B$ star often used as a solar-composition standard.

Because the formerly used standard stars Vega and Sirius (see below) are now felt to have very unusual compositions, and well known stars like $\theta$ Leo and o Peg have been deemed Am, explorations were made by Holweger, Gigas and Steffen (1986) and Holweger, Steffen and Gigas (1986) in the south, and by Ramella et al. (1989) in the north, to search for examples of bright stars near AO with (a) very sharp lines and (b) solar composition, for use as replacement standard stars for differential abundance work. Both groups reported that the compositions of most stars were peculiar in some way. For example, the $\mathrm{C}$ abundance seems very variable. The stars HR 3383 and HR 4138 are ultrasharp lined. Ramella et al. found that, of their 5 stars with $v \sin i<15 \mathrm{~km} / \mathrm{s}$, three are hot Am's, one is a double-lined binary, and the other is probably a variable chemically peculiar SiSr type. They concluded that "...single normal sharplined A0 stars appear to be extremely scanty or non-existent...".

Lemke $(1989,1990)$ analysed the Holweger et al. samples in detail, 
including non-LTE calculations for $\mathrm{He}, \mathrm{Ca}, \mathrm{Fe}$ and $\mathrm{Ba}$ in his work. His conclusions were that most of the observed stars have He spectra consistent with normal $\mathrm{He} / \mathrm{H} \sim 0.10$ but that three stars have low He abundances (HR 3383, HR 4138 and - marginally - Sirius). Also, the $C$ abundance (from $C I$ lines) varies by as much as $1.0 \mathrm{dex}$, as does $\mathrm{Si}$. The $\mathrm{Sr}$ abundance varies enormously (by 1.6 dex) within the sample. The hot-Am signature occurs frequently. My own reading of Lemke's results is that they are in good accord with those of Ramella et al.; solar composition sharp-lined stars near $\mathrm{AO}$ are exceedingly rare and possibly non-existent; perhaps only HR 1975 and $\beta$ PsA are truly normal in this sense, but their vsini's are 33 and $26 \mathrm{~km} / \mathrm{s}$ respectively.

Lemke's results for C are echoed in the studies by Hardorp et al. (1986), who found that the strength variation of the CII resonance lines (1335 $\AA$ ) in IUE spectra gave rise to a large abundance range at types $\mathrm{A} 0-\mathrm{A} 2$, and the same workers found especially strong $\mathrm{C}$ deficiencies in several normal $\mathrm{B}$ stars ( $c f$. Cugier and Hardorp, 1988a, b; Cugier 1989), such as $\alpha$ Leo and $\psi$ Cet. Other stars - $\tau$ Her, $\pi$ Cet and Vega - have essentially solar $C$ abundances. Similar widely variant abundances were found in normal A0 stars by Lambert et al. (1986). Discussion of the reasons for the low $C$ abundances in some stars is beyond the scope of this review.

\section{Vega ( $\alpha$ Lyr) and Possible Vega Analogs}

Vega has a distinctly non-solar composition. The study of Vega continues to be a growth industry, as it challenges us with more and more oddities. Since the discovery of a dust shell (Aumann et al., 1984) around Vega, the suspicion has grown that it may be related to the $\lambda$ Boo stars (reviewed elsewhere).

Gigas (1986) used the Griffins' Vega spectra to study the abundance of Fe using a detailed NLTE calculation. He concluded that $\xi_{t}$ was depthdependent, varying from 1.0 to $2.0 \mathrm{~km} / \mathrm{s}$. The NLTE analysis of FeI yielded an abundance 0.25 dex higher than Fell for the adopted model $\left(\mathrm{T}_{\mathrm{eff}}=9,500 \mathrm{~K}, \log \right.$ $\mathrm{g}=3.9$ ) while in LTE the agreement was essentially perfect. Adelman and Gulliver (1990) used Cowley's Reticon spectra of Vega and found that their equivalent widths were about $10 \%$ weaker than Gigas's. They found (for LTE) $\xi_{\mathrm{t}}=0.6 \mathrm{~km} / \mathrm{s}$ and derived low $\mathrm{He}$ and $\mathrm{C}(\Delta \log \varepsilon=-0.5$ and -0.3 , respectively). Note, however, that Lemke (1990) found Vega to have essentially solar C and $\mathrm{He}$, and Venn and Lambert (1990) found $\mathrm{C}, \mathrm{N}$ and $\mathrm{O}$ to be solar, so these points remain debatable. Stuirenburg and Holweger $(1990,1991)$ found Vega to have $[\mathrm{C} / \mathrm{H}]=-0.30$ in a NLTE analysis. All of the new studies, including that of Savanov and Khalilov (1986), agree that $[\mathrm{Fe} / \mathrm{H}] \approx-0.5$ in Vega, and indeed many other Fe-peak metals are also clearly underabundant by similar amounts, according to the analysis of IUE spectra by Sadakane et al. (1986).

Vega's spectral energy distribution has also been studied. Leggatt (1985) and Mountain et al. (1985), among others, found near-infrared excesses in Vega at wavelengths 3-12 $\mu \mathrm{m}$. Coté (1987) lists several A and B stars with IRAS excess infrared flux; among these are Vega, $\beta$ UMa (A1V) and $\gamma$ Oph (A0V). Knyaseva and Kharitonov (1990) have repeated their work on the absolute energy calibration and find good agreement with Hayes (1985), except in the near UV $(3100-3600 \AA)$ where atmospheric extinction and faintness of lamps are critical problems to overcome. Their absolute fluxes are about $15 \%$ higher at the most extreme short wavelengths observed.

Gulliver et al. (1991) investigated unusual profiles of weak metal lines in 
Vega. The profiles are flat-bottomed rather than Gaussian in shape. The reasons for this are not yet known, but may arise from Vega being a rapid rotator seen nearly pole-on, or they may arise from differential rotation (Elste, 1992).

Vega may well be related to the $\lambda$ Boo stars. Indeed, Baschek and Slettebak (1988) suggested that '...we may regard the $\lambda$ Boo stars as "rotating Vegas" or, conversely, Vega as a mild, non-rotating $\lambda$ Boo star'. Or, Vega could be a rapid rotator seen nearly pole-on. Venn and Lambert (1990) pointed out that $\lambda$ Boo status would suggest that element depletion may proceed by grain condensation processes akin to those suggested as an explanation for abundances in post AGB stars like HD 52961 (Van Winkel et al., 1992). If so, $\mathrm{Zn}$ could provide a crucial test: a near solar abundance for this element would support the grain depletion model. In their study of $\mathrm{Zn}$ in $\mathrm{Hg}-\mathrm{Mn}$ stars, Sadakane $e t$ al. (1988a) found $\mathrm{Zn}$ to be essentially solar in Vega, which was used as one of their comparison stars.

Some A0 stars could be called Vega analogs on the basis of their mild metal deficiencies. Among these are $\alpha$ Dra (A0 III) studied by Adelman et al. (1987), revised by Adelman (1991), and 50 Lib (Lemke 1989, 1990). Gerbaldi and Freire Ferrero (1986) suggested that $40 \mathrm{Cnc}$ is yet another Vega analog, but Pasinetti Fracassini et al. (1990) believe it to be closer to solar composition.

Due to numerous suggestions of possible variability, Ruban (1988) checked records of photometric observations of Vega and concluded that it had been photometrically constant over the range $3,100 \AA-10,000 \AA$ to within \pm 0.01 magnitude for the past 16 years.

\section{HG-MN ABUNDANCES}

A welcome trend noted is for more optical analyses of all types of stars to be done with vastly improved spectra, in the form of photographic coadds, Reticon, or CCD data with high signal-to-noise ratios and high spectral resolution. It is now also far more routine to read papers in which new spectral regions (yellow, red, near infrared) are explored. Those using IUE spectra have published relatively few papers, but at this colloquium Dr K.C. Smith and I summarize in our poster paper some new results from an extensive study of coadded ultraviolet spectra (1300 $\AA-3150 \AA$ ) of $\mathrm{Hg}-\mathrm{Mn}$ and normal stars.

Extremely exciting results have been coming from observations made with the Goddard High Resolution Spectrograph on the Hubble Space Telescope, which Dr D. Leckrone reviews also at this colloquium.

\section{Abundances in Individual Stars}

In a long series of papers, Adelman (1984, 1987, 1988c, 1989; Adelman and Davis Phillip, 1990), presented his photographic LTE fine analyses of $13 \mathrm{Hg}-\mathrm{Mn}$ stars using coadded DAO photographic spectra or (in the most recent work) DAO Reticon spectra. In addition, many participants in the Workshop on Elemental Abundance Analysis (Adelman and Lanz, 1988) studied his coadds of $\varphi$ Her, as did Ryabchikova and Piskunov (1988). As already discussed, Adelman has also analysed several normal or superficially normal comparison stars. The Hg-Mn stars examined by Adelman are: $v$ Her, 38 Dra, 53 Tau, $\mu$ Lep, $\kappa$ Cnc, $\phi$ Her, 28 Her, HR 7664, v Cnc, ı CrB, HR 8349, HR 4817 and $\chi$ 
Lup. It is evident from this painstaking work that most of the previously accepted properties of $\mathrm{Hg}-\mathrm{Mn}$ stars can be confirmed. For example, $[\mathrm{Mn} / \mathrm{H}]$ is strongly correlated with $\mathrm{T}_{\text {eff }}$, but there are occasional severe violations of this trend (e.g., HR 7664). The abundance of Fe ranges over more than an order of magnitude (53 Tau is Fe weak; HR 7664 is Fe strong). Both $\mathrm{Sr}$ and $\mathrm{Y}$ are overabundant in nearly all $\mathrm{Hg}-\mathrm{Mn}$ stars, but $\mathrm{Zr}$ is very strong in some stars and undetected in others.

Dobrichev et al. (1989a) analysed the binary HR 4072, deriving $\mathrm{T}_{\text {eff }}=$ $10,100 \mathrm{~K}$. Lopez-Garcia and Gargiulo (1989) analysed HD 161698 in IC 4665, describing it as a typical Hg-Mn star. However, the analysis was based on rather low dispersion and should be regarded as preliminary. Lyubimkov and Polosukhina (1988) performed an abundance analysis of $\alpha$ And. They noted a difficulty in reconciling Fel/FelI ionization ratios and $\mathrm{T}_{\text {eff }}$ from photometric criteria. Zöchling and Muthsam (1987) discussed their analysis of $\kappa$ Cnc based on Cowley's DAO spectra, and on model atmospheres with a high (selfconsistent) $\mathrm{Mn}$ abundance incorporated in the opacity. These authors showed that the flux they predict in the ultraviolet agrees with observations far better than predictions based on the solar composition.

\section{Abundance Studies of Individual Elements}

He: Wolff and Heasley (1985) surveyed He abundances in normal main sequence B stars, mostly those hotter than $\mathrm{Hg}-\mathrm{Mn}$ stars, down to $\mathrm{T}_{\text {eff }}=15,000 \mathrm{~K}$. This paper might serve as a good starting point for other investigators. They deduced an average $\log \mathrm{N}(\mathrm{He}) / \mathrm{N}(\mathrm{H})=-1.06$. Adelman found similar values for late $\mathrm{B}$ normal stars in his series of papers. However, all $\mathrm{Hg}-\mathrm{Mn}$ stars he studied (see above) had low He abundances, by between 0.5 and $1.2 \mathrm{dex}$. Dobrichev et al. $(1989 \mathrm{~b}$ ) found $\mathrm{He} / \mathrm{H}=0.017$ in $\mathrm{K}$ Cnc, while Adelman's (1989) result is 0.0055 . This difference seems too large to be accounted for by different adopted model atmosphere parameters or analysed lines. Dobrichev et al. also found definite evidence for a high $3 \mathrm{He} / 4 \mathrm{He}$ ratio $(0.35)$, which had previously only been suspected by Hartoog and Cowley (1979).

Be and B: Sadakane et al. (1985) extended earlier studies of the resonance lines of these elements to a sample of $23 \mathrm{Hg}-\mathrm{Mn}$ stars. Boron (BII $1362.46 \AA$ ) is strong in $\sim 40 \%$ of the $\mathrm{Hg}$-Mn sample stars; Be is strong in $\sim 65 \%$ (Bell 3130,31 $\AA)$. All possible combinations of strong/weak $B / B e$ occur in their sample. The absence of the BII line indicates underabundance in most cases.

Earlier ground-based work by Boesgaard et al. (1982) had suggested a strong $\mathrm{T}_{\text {eff }}$ dependence of Be overabundances, which was noted by Borsenberger et al. (1984) in their theoretical discussion. Smith (1992) has re-analysed by spectrum synthesis techniques what is essentially the sample observed by Sadakane et al. (1985). His results are summarized in the histogram of Fig. 1 . There is no obvious $T_{\text {eff }}$ trend in this sample. This suggests that the trend seen by Boesgaard et al. was due to a small sample size coupled with some bad luck in not happening to include in their work three Be-strong cool $\mathrm{Hg}-\mathrm{Mn}$ stars. The suggested mechanism for creating a temperature trend (Borsenberger et al., 1984), which involves ad hoc thickening of the He II convection zone, is probably no longer necessary.

C. N and O: Dworetsky (1986) noted the absence of studies of CNO systematics in Hg-Mn stars compared to normal stars. The literature now contains many excellent attacks on these important elements. Normal stars have already been 


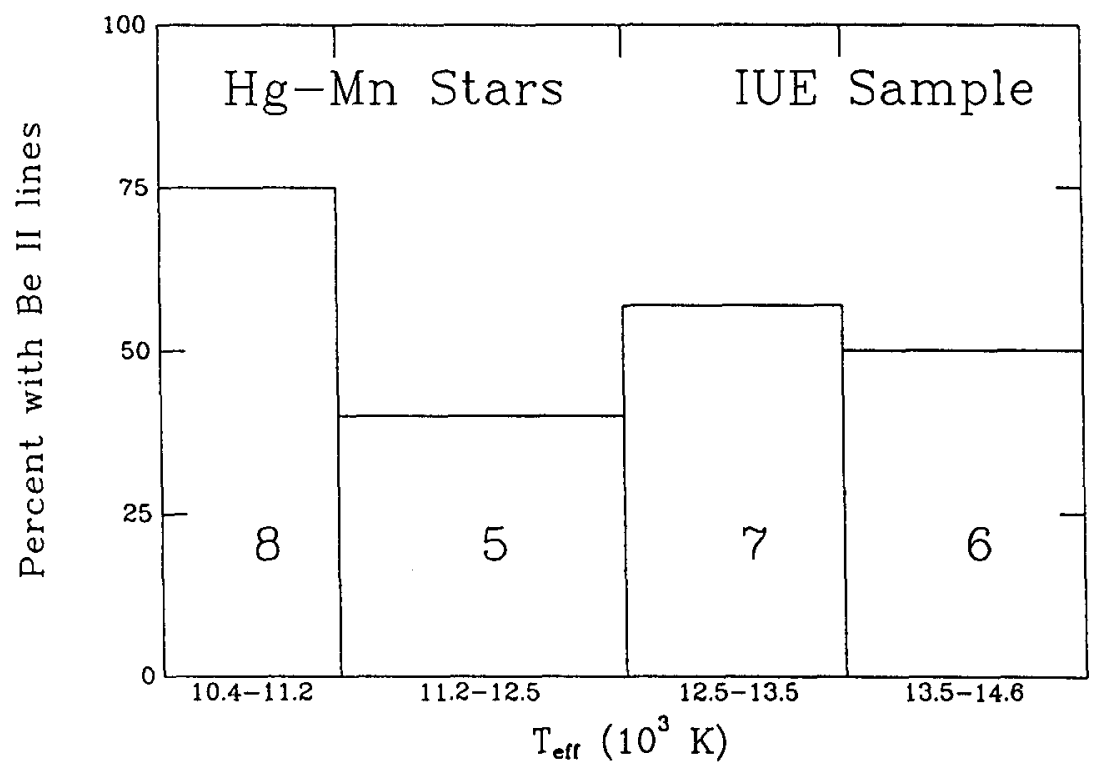

Fig. 1. Histogram showing the fraction of $\mathrm{Hg}-\mathrm{Mn}$ stars with strong BeII resonance lines in the IUE sample studied by Smith (1992) as a function of temperature. The numbers in each range are shown in the boxes.

There is no trend, in contradiction to Boesgaard et al. (1982).

discussed above. Lyubimkov (1989) discussed NLTE calculations for CII 1335 $\AA$ and $4267 \AA$, mainly with applications to B stars hotter than most Hg-Mn stars. Roby and Lambert (1990) studied optical CI and NI while Leushin et al. (1990) examined CII and NII for abundances in relatively small samples. Roby and Lambert found $\mathrm{C}$ to have approximately normal abundance, but $\mathrm{N}$ was notably deficient and $\mathrm{O}$ mildly deficient in $\mathrm{Hg}-\mathrm{Mn}$ stars. Leushin et al. found different results altogether. Roby et al. (1989) had previously found $\mathrm{N}$ to be very underabundant ( $\sim 2.0 \mathrm{dex}$ ) in two Hg-Mn stars. Lanz and Artru (1990) also examined $\mathrm{N}$ in IUE spectra of $\mathrm{Hg}-\mathrm{Mn}$ stars and concluded it was very underabundant compared to normal stars. Takada-Hidai (1990) examined infrared NI lines in the region $8629 \AA-8729 \AA$ (the same lines examined by Roby and Lambert) and derived a strong underabundance $\geq 1.0$ dex in three $\mathrm{Hg}-\mathrm{Mn}$ stars, but found found essentially solar abundances in the comparison stars. Smith and Dworetsky (1990a) surveyed the CII $1335 \AA$ resonance lines, and NI lines at $1492 \AA, 1494 \AA, 1742 \AA$ and $1745 \AA$, in a sample of 14 normal and $24 \mathrm{Hg}-\mathrm{Mn}$ stars, using coadded IUE spectra. While most $\mathrm{Hg}-\mathrm{Mn}$ stars in that study were found to have essentially normal $C$ abundances, or are slightly underabundant, three exhibited significant 1-2 dex underabundances of $C$ : $H R$ $7664,46 \mathrm{Aql}$, and $112 \mathrm{Her}$. Smith and Dworetsky also found that $\mathrm{N}$ seems to be universally underabundant in $\mathrm{Hg}-\mathrm{Mn}$ stars, by at least $1.0 \mathrm{dex}$ in most cases, with respect to their normal star sample, for which the mean derived abundance is essentially solar. Thus, the results from ground-based and ultraviolet observations of $\mathrm{N}$ are in complete agreement. Oxygen is very difficult to study in the form of OlI because all the optical lines are very weak. Adelman (1989) 
found evidence for OII in HR 8349, but the derived abundance seems rather high and there may be some doubt concerning the identification. The strong OI triplet at $7773 \AA$ has long been known as a potential source of $\mathrm{O}$ abundances in A and B stars (van Santvoort, 1987). An extensive survey of normal and CP stars was undertaken by Faraggiana et al. (1988) and Gerbaldi et al. (1989). Once NLTE effects were taken into account, normal stars gave essentially solar abundances, while most $\mathrm{Hg}-\mathrm{Mn}$ stars showed a deficiency of order 0.5 dex. A small number of $\mathrm{Hg}-\mathrm{Mn}$ stars seem to have normal $\mathrm{O}$ abundances.

Fe-Peak Elements: The only cross-sectional survey of abundances published up to the end of 1991 was that by Smith and Dworetsky (1990b), which is described more fully by Smith (1992) and which will be published in more detail elsewhere. In that study, $26 \mathrm{Hg}-\mathrm{Mn}$ stars were compared differentially with 14 normal stars using coadded archival IUE high resolution spectra. Results were given for $\mathrm{Cr}, \mathrm{Mn}, \mathrm{Fe}, \mathrm{Co}$ and $\mathrm{Ni}$. In most cases the normal comparison stars give approximately solar abundances. In the case of $\mathrm{Fe}$, their original statement that the normal stars seemed slightly underabundant must now be modified to take into account the "new" solar abundance $\left(\log \mathrm{N}_{\mathrm{Fe}}=7.50\right.$ ), which agrees well with the UV results, except for Vega, in which the UV lines yield abundances from FeII which agree closely with those of Gigas (1986) and other recent investigations.

The $\mathrm{Cr}$ abundances in $\mathrm{Hg}-\mathrm{Mn}$ stars range over 2.3 dex in the studied sample, far more than can be accounted for by errors in the analysis. The cooler stars have roughly solar abundances, but several stars with $T_{\text {eff }}>13,000 \mathrm{~K}$ have deficiencies of order $1.5 \mathrm{dex}$. Mn was found to have a clearly delineated upper envelope of abundances which ranges from approximately solar at $10,000 \mathrm{~K}$ to 2.6 dex overabundance at $15,000 \mathrm{~K}$. This result agrees with Adelman's (1989) result for 9 stars, even including the fact that there are stars which tend to have only mild $\mathrm{Mn}$ enhancements, and which fall below the envelope in an abundance-temperature plot. The 1.5 dex scatter of Fe abundances is real. The hotter stars tend to be the most Fe-rich. The behaviour of Co was shown to be very odd, reminiscent of B. Most $\mathrm{Hg}-\mathrm{Mn}$ stars are at least 2.0 dex underabundant in $\mathrm{Co}$, with a few Co-rich exceptions like $\nu \mathrm{Cnc}$ and $\varphi$ Her. This discussion provided the first systematic determination of Co abundances in $\mathrm{Hg}$ $\mathrm{Mn}$ stars. Finally, most $\mathrm{Hg}-\mathrm{Mn}$ stars seem to be significantly Ni-deficient, with v Cnc being an exception.

$\mathrm{Zn}$ : Sadakane et al. (1988a) studied the ZnIl resonance line at $2062 \AA$ in a survey of $23 \mathrm{Hg}-\mathrm{Mn}$ and 6 normal stars. (The other line, $2025 \AA$, is affected by blending.) There is no obvious pattern in $\mathrm{Zn}$ abundances: relative to the standard stars, there are five $\mathrm{Hg}-\mathrm{Mn}$ stars with $\mathrm{Zn}$ enhancements of +1.0 dex or more, and four detections with essentially solar abundances; the remaining stars yield only upper limits of about -1.0 dex depletion. The behaviour of $\mathrm{Zn}$ resembles closely that of $\mathrm{Co}$, as described above.

$\mathrm{Ga}$ : Takada-Hidai et al. (1986) examined the systematics of GaII (1414 $\AA$ ) and GaIII (1495 $\AA$ ) in Hg-Mn, He-weak, Si (magnetic) and normal stars. In Hg-Mn stars, 15 of the 20 hottest stars showed strong enhancements of 2.6-3.8 dex, while amongst the cooler Hg-Mn stars only HR 7775 showed a significant enhancement, according to this study. However, these authors pointed out that much of the difficulty and loss of sensitivity they had in analysing Ga was due to the existence of an unidentified blend in some stars just shortward of the central wavelength they used. Around the same time, Isberg and Litzén $(1985,1986)$ 
published their study of the spectra of GaII and GaIII, giving revised wavelengths which match perfectly the "blend" feature. In other words, there is no blend; the line seen is in fact mostly Gall. This identification produces an entirely new situation; the problem should be re-examined. It seems likely (Smith, 1992) that all $\mathrm{Hg}-\mathrm{Mn}$ stars have enhanced $\mathrm{Ga}$ abundances.

In other developments, Lanz et al. (1990) examined the GaII $6334 \AA$ line instead of the blue lines, which arise from very high excitation levels. This line is much easier to detect and may make it possible to do deeper surveys.

The GaII resonance line is so strong when $\mathrm{Ga}$ is overabundant that it can be detected easily in low resolution IUE spectra. Jaschek and Jaschek (1987) have found it in a number of stars surveyed, including $\mathrm{Hg}-\mathrm{Mn}, \mathrm{Si}-4200$ and $\mathrm{He}-$ weak stars. But they also found $\mathrm{Ga}$ in the spectra of a few stars otherwise classified as normal, with no obvious optical peculiarity.

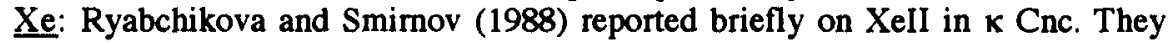
presented new oscillator strengths in that paper.

$\mathrm{Pt}, \mathrm{Au}$ and $\mathrm{Hg}$ : The problem of $\mathrm{Pt}$ abundances and isotopes in $\mathrm{Hg}-\mathrm{Mn}$ stars is a longstanding one. Until very recently there was no extant study of isotopic wavelength shifts in PtII, although such shifts have long been suspected in stars from studies of $\chi$ Lup, HR 4072 and other stars (Dworetsky and Vaughan, 1973; Dworetsky, 1969). Engleman (1989) has presented full and complete experimental measurements of PtII isotopic shifts and hyperfine structure in all the prominent optical lines seen in $\mathrm{Hg}-\mathrm{Mn}$ stars. This work confirms the proposal that $\mathrm{Pt}$ in $\chi$ Lup is dominated by ${ }^{198 \mathrm{Pt}}$, the heaviest isotope. It is hoped that new observations will be stimulated by this news. Further work on the strong ultraviolet lines is also needed. Fuhrmann (1989) has presented convincing evidence for the presence of AuII as well as PtII and HgII in the ultraviolet spectra of several Hg-Mn stars. This accords well with the previous identification of AuIl in the optical spectrum of HR 7775, where it is very evident, and in $\chi$ Lup and HR 4072 (Guthrie, 1985). Wahlgren et al. (1992) report observations of AuII with the GHRS on the HST in $\chi$ Lup and $\kappa$ Cnc. Note that isotopic studies are not needed as Au has only one stable isotope, 197Au. Leckrone et al. (1991) reported that the first observation made with the GHRS was of the HgII $1942 \AA$ resonance line in $\chi$ Lup. Dr Leckrone will discuss these results in detail in his paper. This observation confirmed the longstanding result that $204 \mathrm{Hg}$ dominates in this star, indicating that a high degree of fractionation has taken place $(q=3.0)$.

\section{RELATED STARS}

\section{Sirius ( $\alpha \mathrm{CMa}$ )}

I have already mentioned Lemke's $(1989,1990)$ examination of Sirius; he found that He was marginally underabundant, that $C$ is clearly depleted by about 0.6 dex and that $\mathrm{Fe}$ is enhanced by $0.3 \mathrm{dex}$. The heavier elements $\mathrm{Sr}$ and $\mathrm{Ba}$ have abundances up to 1.0 dex above solar. These characteristics confirm that Sirius has properties we usually describe as "hot Am" and should be considered a member of that class. Savanov (1987) confirmed this view (except that Ca and Sc were reported to be normal). He found a generally increasing enhancement of abundances with atomic number for $\mathrm{Fe}$ and elements such as rare earths, $\mathrm{Sr}$ and Ba. Sadakane and Ueta (1989) examined the region $3530 \AA-4410 \AA$ and confirmed the high Fe abundance, and the overabundance of $\mathrm{Sr}, \mathrm{Y}$ and $\mathrm{Zr}$ by 
about 0.9 dex, but found $\mathrm{Ca}$ and $\mathrm{Sc}$ to be strongly depleted (by 0.6 and 1.0 dex respectively). Sadakane et al. (1988b) examined Copernicus ultraviolet spectra and found that a weak HgII $1942 \AA$ resonance feature was present, indicating an abundance about 1.3 dex above the solar system value. Recently, Sadakane (1991) has also searched for other heavy elements and will review this work at this colloquium. The Griffins (1985) looked closely at the BeII resonance lines at 3130-31 $\AA$ and found no trace of the lines present, indicating depletion of 1.0 dex or more.

\section{Other Related Stars}

The magnetic star $33 \mathrm{Gem}$ (Glagolevskij et al., 1985) is unique. It is apparently a magnetic variable, although the spectrum does not vary much. It has a composition like a $\mathrm{Hg}-\mathrm{Mn}$ star (high $\mathrm{Be}, \mathrm{P}, \mathrm{Mn}, \mathrm{Ga}$, maybe $\mathrm{Hg}$; low $\mathrm{N}, \mathrm{Co}$ and $\mathrm{Zn}$ ) and does not fit any of the usual magnetic composition groups. If nothing else, the existence of $33 \mathrm{Gem}$ tells us to be wary of completely separating the magnetic and non-magnetic peculiar stars as distinct phenomena. Population II stars sometimes have characteristics similar to $\mathrm{Hg}-\mathrm{Mn}$ or He-weak stars like 3 Cen A. CL 1083 in NGC 6752 has $\mathrm{T}_{\text {eff }}=16,000 \mathrm{~K}, \log \mathrm{g}=4.0, v \sin i<15$ $\mathrm{km} / \mathrm{s}$, low He abundance, and high $\mathrm{Fe}$ abundance (Glaspey et al., 1989). Klochkova (1985) found two probable $\mathrm{Hg}$ stars in the Orion OB1 association, HD35548 and HD36549. This is another indication of the rapidity with which chemically peculiar characteristics can appear in newly formed stars.

\section{MAGNETIC FIELDS}

Glagolevkij et al. (1989) and Bychkov et al. (1990) summarized their work on stellar magnetic fields. They found no fields (upper limits 10-15 G) in $\alpha$ Lyr, $\alpha$ $\mathrm{CMa}$, and $\alpha \mathrm{Gem}$, nor were fields detected down to the similar limits of their instruments for the Hg-Mn stars $\alpha$ And, $\mu$ Lep, $\varphi$ Her, ı CrB, HR 4072, or $v$ Her. Dipolar or other ordered fields are probably less than 15 gauss in $\mathrm{Hg}-\mathrm{Mn}$ stars. On the other hand, $33 \mathrm{Gem}$ was reported to have a strong field.

Recent work by Mathys and Lanz (1990) suggests that certain stars may have strong disordered (non-dipolar) fields, rather like the Sun, so that overall a Zeeman shift would not be detected spectroscopically, but spectral lines might be modified in strength by desaturation mechanisms. Two methods, the StenfloLindegren (1977) technique and the partial Paschen-Back effect on a close pair of equal-intensity lines, were tested on the hot Am star o Peg, with apparent detection of a field of order $2 \mathrm{kG}$. Takada-Hidai and Jugaku (1992) attempted to use the second method to look for a field in the Hg-Mn star $\mu$ Lep, but were thwarted because the line pair being used, FeII $(6147 \AA, 6149 \AA)$, is blended with $\mathrm{HgIl} 6149.5 \AA$. This will make it very difficult to apply the method to any Hg-Mn star. Perhaps the Stenflo-Lindegren technique is more promising (Lebedev, 1990).

\section{PHOTOMETRIC VARIABILITY IN HG-MN STARS}

Catalano et al. (1988) found light variations of small amplitude in 53 Tau, with a period close to, but not identical with, the known orbital period of this spectroscopic binary. Schneider (1987) published additional observations of low amplitude periodic variations of order several days in $\mathrm{Hg}-\mathrm{Mn}$ stars. In 
general, these do not correspond to the known binary periods.

McNamara (1987) searched for short-period microvariability in Pleiades B stars. Although some do vary, the star 20 Tau (Maia), which seems to be closely related to $\mathrm{Hg}-\mathrm{Mn}$ stars, showed no significant variability at the limit of detection, 2 mmag.

Zverko et al. (1987) found some very odd variations in the CaII $\mathrm{K}$ line of $\alpha$ And. While the Balmer lines showed no sign of variability over several hours, the $\mathrm{K}$ line oscillated over a range of $9 \mathrm{~km} / \mathrm{s}$ on time scales of a few minutes. Further observations are urgently needed; if this is real, we certainly do not understand these stars!

\section{LINE IDENTIFICATION}

There were very few papers addressing this problem specifically. Ramella et al. (1987) discussed line identification in the ultraviolet for solar composition B2B9.5 stars. Guthrie (1985) found evidence for AuII, PrII, NdII, and GaI in optical Hg-Mn spectra. Mathys and Cowley (1992) discussed PrIII in magnetic Ap stars and remind us that this species was originally identified in $\chi$ Lup in the 1960s. Adelman (1989) has found some evidence for weak GdII in three HgMn stars. Ansari (1987) has applied an extension of wavelength coincidence statistics (WCS) in which lines are grouped by predicted strength. This may lead to improvements in abundance estimates made from WCS studies.

\section{BINARITY AND ROTATION}

An excellent overview of the statistics of binary stars among $\mathrm{Hg}-\mathrm{Mn}$ stars was given by Gerbaldi et al. (1985). The $\mathrm{Hg}-\mathrm{Mn}$ stars (54\%) have an essentially normal binary frequency ( $47 \pm 5 \%$ for normal stars) but an unusually high proportion of double-lined binaries. No $\mathrm{Hg}-\mathrm{Mn}$ star has a binary period less than three days, so it is not surprising that there is only one known eclipsing binary $\mathrm{Hg}-\mathrm{Mn}$ star. There is at first sight a preponderance of high-eccentricity orbits, but when one excludes eclipsing binaries from the normal star sample the remaining eccentricities are similarly distributed.

Guthrie (1986) summarized the available evidence on inclinations of late B-type spectroscopic binaries and concluded that the inclination distribution for $\mathrm{Hg}-\mathrm{Mn}$ binaries in double-lined systems is consistent with random orientation. He listed several examples of systems in which the primary stars are rotating subsynchronously, that is, vsin $i$ is much smaller than expected for synchronous rotation with $\mathrm{P}($ spin $)=\mathrm{P}$ (orbit), assuming that the spin axes are aligned perpendicular to the orbit plane. The primaries of HR266, HR4072, $\chi$ Lup and 74 Aqr were singled out. More recently, Baade (1989a, b) observed 74 Aqr and reported $v \sin i<5 \mathrm{~km} / \mathrm{s}$, making this entry on Guthrie's list even more anomalous. The metal-weak A0III star HR7338 is also subsynchronous. But, the system 74 Aqr is even more remarkable than the others because its period of 3.43 day is one of the shortest known for a Hg-Mn star!

Klochkova and Kopylov (1985) presented a summary of their investigations of the evolution of rotation of various types of peculiar stars in open clusters and associations. In their work, they removed effects of cluster-to- 
cluster angular momentum distribution variation by normalizing to the mean $v$ sini of each cluster's normal stars. They then looked for evolutionary changes of peculiar-star rotation with age. They found no significant trends for any types; $\mathrm{Hg}-\mathrm{Mn}$ stars, as a group, have the lowest mean $v \sin i$ of any stars studied.

It may not be long before we can investigate some of these binaries another way. Pan et al. (1992) have obtained the visual orbit of $\alpha$ And, using the Mark III Stellar Interferometer on Mount Wilson, California. They were able to derive the inclination directly, but not the mass ratio. However, they found an accurate estimate of $\Delta \mathrm{m}=2.01 \mathrm{mag}$, which explains why recent searches for the secondary spectrum have proved difficult.

\section{TEMPERATURE AND GRAVITY CALIBRATIONS}

Several useful and important calibrations have been published since 1985 which greatly help us to determine accurate $\mathrm{T}_{\text {eff }}$ and $\log \mathrm{g}$ for both normal and $\mathrm{Hg}-\mathrm{Mn}$ stars. Much of this work was put into perspective in Elemental Abundance Analyses (Dworetsky, 1988). The observations available are usually StrömgrenCrawford uvby $\beta$ indices or Geneva $X, Y$ parameters. If adequate spectra are available one may also have Balmer line profiles, and if someone has gone to enough trouble one may be fortunate enough to have spectrophotometry. If one is very fortunate, ultraviolet spectrophotometry will also exist. The only question remaining is, how does one transform from the observations to accurate (as opposed to precise) $\mathrm{T}_{\text {eff }}$ and $\log \mathrm{g}$ ?

Moon and Dworetsky (1985; MD 85) used a semi-empirical method to transform the theoretical $c_{o}, m_{O}$, (b-y) values of Relyea and Kurucz (1978), combined with theoretical $\beta$ indices, into grids which matched fundamentallydetermined $\mathrm{T}_{\text {eff }}$ 's, and matched gravities for selected binary stars; see also Moon (1985) and Dworetsky and Moon (1986). A similar goal was reached by Lester, Gray and Kurucz (1986; LGK) who adopted $\mathrm{T}_{\text {eff }}$ and $\log \mathrm{g}$ for five standard stars whose temperatures were determined from spectrophotometric fits of model atmospheres. The gravities were, with one exception, fundamental in the sense that they made use of the mass-luminosity relationship and accurate parallax information.

Two tests comparing LGK and MD85 have been carried out recently. In both cases the authors were partly motivated to extend the grids to higher $T_{\text {eff's }}$ than originally intended. Castelli (1991) found that the two calibrations differ by about $125 \mathrm{~K}$ near $10,000 \mathrm{~K}$, and by nearly $500 \mathrm{~K}$ near $20,000 \mathrm{~K}$ (LGK temperatures are lower); the LGK grid produces smaller gravities, by about 0.25 dex, at $20,000 \mathrm{~K}$. Both gravity calibrations agree closely at $10,000 \mathrm{~K}$. Castelli

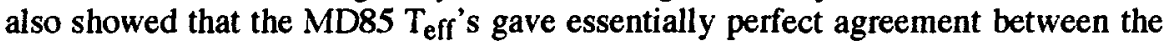
$\mathrm{T}_{\text {eff's }}$ ' Morossi and Malagnini (1985) and MD85 for values up to $18,000 \mathrm{~K}$. The second test was carried out by Napiwotzki et al. (1992). They used bolometric-flux fundamental $\mathrm{T}_{\text {eff's }}$ and, for hotter stars $(>8,500 \mathrm{~K})$, checked the gravity calibrations of MD85 and LGK against CCD observations of Balmer line profiles. The MD85 calibration agreed with the Balmer line results within 0.07 dex for the hotter stars and within 0.04 dex for the intermediate temperature $\left(8,500 \mathrm{~K}<\mathrm{T}_{\text {eff }}<11,000 \mathrm{~K}\right)$ stars. They found (as did Castelli) that the LGK gravity calibration deviates strongly at temperatures around $20,000 \mathrm{~K}$ (but by up to $0.4 \mathrm{dex}$ ). They also found systematic problems with $\mathrm{T}_{\mathrm{eff}}$ similar to those 
noted by Castelli.

The source of the differences between MD85 and LGK is undoubtedly in the choice of standard stars. LGK used only one B star ( $\eta$ UMa, B3V) to calibrate their grids and it is neither a fundamental star for gravity nor a firstclass uvby $\beta$ standard. All their other standards were of class A0 or later. Napiwotzki et al. suggest that adoption of too low a gravity for $\eta$ UMa by LGK could be in part the source of the differences seen.

North and Kroll (1989) compared Balmer line profiles with uvby $\beta$ and Geneva photometry calibrations of gravity for Ap stars. For normal and Hg-Mn stars, all three methods were in good agreement.

North and Nicolet (1990) have obtained an important new calibration of Geneva photometry (X, $\mathrm{Y}$ parameters) in terms of $\mathrm{T}_{\mathrm{eff}}$ and $\log \mathrm{g}$. They adopted the semi-empirical approach used by MD85, using many of the same standards, and the two calibrations are in good agreement. Hauck and Slettebak (1989) showed that $(X, Y)$ are insensitive to rotational-velocity effects.

Gulyayev et al. (1987) discussed some new methods for deducing $\mathrm{T}_{\text {eff }}$

and $\log g$ with parameters based on flux measurements at $3687 \AA, 3812 \AA$ and $3862 \AA$. Their parameters $\mathrm{K}, \mathrm{M}, \mathrm{N}$ and $\mathrm{L}$ form a more or less orthogonal set in the $\mathrm{T}_{\text {eff }}-\log \mathrm{g}$ plane. The results are usually consistent, but are they accurate? There are some very promising possibilities for further work here.

Kopylov et al. (1989a,b) described their extensive work on quantitative line-intensity spectral classification. These methods permit the authors to discern between, for example, "early" and "late" B7 stars, and also to parameterize chemically peculiar stars of all types. It would be interesting to see how their methods conf ront uvby $\beta$ or Geneva photometry.

I wish to mention also the work of Malagnini and Morossi (1990), who have used flux distributions and parallaxes to deduce $\mathrm{T}_{\text {eff }}$ and $\mathrm{L}_{\mathrm{bol}}$ for many bright stars, a few of which are late B-A0. A good estimate of the stellar mass quickly leads to reasonably accurate gravities.

\section{CONCLUDING REMARKS}

It is gratifying to have seen substantial progress on the points raised in the concluding remarks of the previous review, but new questions arise as old ones are answered. The study of $\mathrm{CNO}$ abundances in A and B stars has come a long way since 1985 . Through the efforts of many workers and research groups, the systematics of abundances in $\mathrm{Hg}-\mathrm{Mn}$ stars are being explored, but it is evident from the work of the Japanese, Franco-Italian and United Kingdom groups, and from Adelman's analyses, that a piecemeal approach of analysing small numbers of stars does not define the phenomenon very well. Cross-sectional studies of larger samples of stars are showing us that the $\mathrm{Hg}-\mathrm{Mn}$ phenomenon is heterogeneous, i.e., within the overall class there are subgroups such as the Cweak Hg-Mn stars, the Co-rich stars, etc. It is only by studying these larger samples of statistically significant size that we can come to grips with the full complexity of the situation we are now confronting. Please be sure to quote me in your next application for telescope time!

The exploration of the boundary region between $\mathrm{Hg}-\mathrm{Mn}$ stars and hot Am stars is a fascinating exercise and seems to be well underway. Is it not remarkable that we find a few solar-composition late B stars like $v$ Cap co- 
existing with cool $\mathrm{Hg}-\mathrm{Mn}$ stars like $v \mathrm{Cnc}$ and $\mathrm{HR} 4072$ at very nearly the same

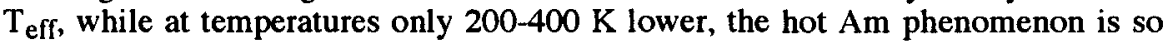
widespread that apparently we have yet to discover a sharp-lined solar composition A0 star? And curious facts are emerging about the brightest star in the sky (excluding the Sun). Sirius lies just to the cool side of the Hg-Mn stars in the HR diagram, and it fits in comfortably with the hot Am stars that inhabit the region very close to $10,000 \mathrm{~K}$. Except for spectacular $\mathrm{Hg}$ and Pt excesses, which have not been found in hot Am's, the remaining abundances in these stars would typically feel right at home in a cool $\mathrm{Hg}-\mathrm{Mn}$ star.

There are now much better tools available for determining $\mathrm{T}_{\mathrm{eff}}$ and $\log \mathrm{g}$ for mid-B to A0 stars in the form of uvby $\beta$ and Geneva calibrations. These are proving useful in every aspect of our work. The use of $T_{\text {eff }}-\log g$ plots to compare different observational criteria for individual stars is a common practice, and a good one in my opinion, but Dr Gigas' calculations of NLTE in Fe suggest that one should abandon the use of ionization balance (in LTE calculations) as part of this exercise, if such was previous practice.

I wish to insert a brief technical point about surface gravity. Adelman's analyses confirm that $\mathrm{He}$ abundances are low in $\mathrm{Hg}-\mathrm{Mn}$ stars. However, a long time ago Auer et al. (1966) demonstrated that one could correctly analyse abundances relative to $\mathrm{H}$ in a $\mathrm{He}$-poor $\mathrm{Hg}-\mathrm{Mn}$ star using a solar $\mathrm{He} / \mathrm{H}$ model, but that a model with slightly lowered surface gravity would necessarily be deduced for it from spectrophotometry and Balmer line profiles. Smith (1992) has validated Auer et al.'s formula for $\Delta \mathrm{g} / \mathrm{g}$ for the $\mathrm{T}_{\mathrm{eff}}$ range of $\mathrm{Hg}-\mathrm{Mn}$ stars. (At slightly lower temperatures it is no longer valid to use their formulation.) If one wishes to calculate $\mathrm{Hg}-\mathrm{Mn}$ abundances, the model with normal He and lower gravity may be applied with insignificant error on $[\mathrm{M} / \mathrm{H}]$, but if one wishes to examine the evolutionary status of the $\mathrm{Hg}-\mathrm{Mn}$ star, due allowance for this effect should be made.

We have seen some of the fruits of our efforts to engage the attention of the atomic spectroscopists to aid our cause. New line identifications, improved wavelengths, and new studies of isotopic structure have helped enormously, and of course the continuing improvement of oscillator strengths and line broadening coefficients, from both experiment and theory, has profound implications for our ability to do new science. If you want to recruit one of these people to help us, the surest way to provoke their interest is to show them the spectrum of an ultra-sharp-lined Hg-Mn star!

From the point of view of "other" physics, I am increasingly persuaded that the great mystery of the origin of $\mathrm{Hg}-\mathrm{Mn}$ stars and related objects is concerned with the reasons for their slow rotation. The 3.43 day binary 74 Aqr is a case in point. One can see from Baade's spectra that both components are very slow rotators, as the MgII $4481 \AA$ lines are cleanly split in each. One might ordinarily expect synchronous rotation to have developed during contraction to the main sequence, if not afterwards. I am convinced that stars like 74 Aqr are telling us something quite fundamental about stellar interiors and evolution, and about star formation. I hope some theoreticians will take up this most curious problem. 


\section{REFERENCES}

Adelman, S.J. 1984, Astr. Ap. Suppl., 58, 585.

Adelman, S.J. 1986, Astr. Ap. Suppl., 64, 173.

Adelman, S.J. 1987, MNRAS, 228, 573.

Adelman, S.J. 1988a, MNRAS, 230, 671.

Adelman, S.J. 1988b, MNRAS, 235, 749.

Adelman, S.J. 1988c, MNRAS, 235, 763.

Adelman, S.J. 1989, MNRAS, 239, 487.

Adelman, S.J. 1991, MNRAS, 252, 116.

Adelman, S.J., Bolcal, C., Koçer, D. and Inelman, E. 1987, PASP, 99, 130.

Adelman, S.J. and Davis Phillip, A.G. 1990, Astr. Ap., 238, 111.

Adelman, S.J. and Fuhr, J.R. 1985, Astr. Ap., 152, 434.

Adelman, S.J. and Gulliver, A.F. 1990, Ap. J., 348, 712.

Adelman, S.J. and Lanz, T. 1988, Elemental Abundance Analyses

(Institut d'Astronomie, Univ. de Lausanne)

Anders, E. and Grevess, N. 1989, Geochim. Cosmochim. Acta, 53, 197.

Ansari, S.G. 1987, Astr. Ap., 181, 328

Auer, L.H. Mihalas, D., Aller, L.H. and Ross, J.E. 1966, Ap. J., 145, 153.

Aumann, H.H., Gillett, F.C. Beichman, C.A., de Jong, T., Houck, J.R., Low, F.J., Neugebauer, G., Walker, R.G. and Wesselius, P.R., 1984, Ap. J. 278, L23.

Baade, D. 1989a, Astr. Ap. Suppl., 79, 42.

Baade, D. 1989b, Astr. Ap., 222, 200.

Baschek, B. and Slettebak, A. 1988, Astr. Ap., 207, 112.

Biémont, E., Badoux, M., Kurucz, R.L., Ansbacher, W. and

Pinnington, E.N. 1991, Astr. Ap., 249, 539.

Boesgaard, A.M., Heacox, W.D., Wolff, S.C., Borsenberger, J. and Praderie, F. 1982, Ap. J., 259, 723.

Booth, A.J. 1989, Astr. Ap., 208, 287.

Borsenberger, J., Michaud, G. and Praderie, F. 1984, Astr. Ap., 139, 147.

Bychkov, V.D., Glagolevskij, Ya, V., El'kin, V.G., Kopylova, F.G., Najdenov,

I.D., Romanyuk, I.I., Churnakova, N.M. and Shtol', V.G.

1990, Astrofiz. Issled. Izv. Spets. Astrofiz. Obs., 30, 78.

Castelli, F., 1991, Astr. Ap., 251, 106.

Catalano, F.A., Leone, F. and Vaccari, S. 1988, I.B.V.S. No.3220

Coté, J. 1987, Astr. Ap., 181, 77.

Cugier, H. 1989, Astr. Ap., 214, 168.

Cugier, H. and Hardorp, J. 1988a, Astr. Ap. 197, 163.

Cugier, H. and Hardorp, J. 1988b, Astr. Ap., 202, 101.

Dobrichev, V.M., Klochkova, V.G., Panchuk, V.E. and Raikova, D.V.1989a, Astrofiz. Issled. Izv. Spets. Astrofiz. Obs. 27, 3.

Dobrichev, V.M., Rajkova, D.V., Ryabchikova, T.A. and Topil'skaya, V.M., 1989b, Astrofizika, 30, 91.

Dobrichev, V.M., Ryabchikova, T.A. and Raikova, D.V. 1986, Astrofizika, 26, 55.

Dworetsky, M.M. 1969, Ap. J. 156, L101.

Dworetsky, M.M. and Vaughan, Jr., A.H. 1973, Ap.J., 181, 811.

Dworetsky, M.M. 1986, in Upper Main Sequence Stars with Anomalous Abundances, (IAU Colloq. No. 90, D. Reidel), ed. C.R. Cowley, M.M. Dworetsky, C. Mégessier, p.397. 
Dworetsky, M.M. and Moon, T.T. 1986, MNRAS, 220, 787.

Dworetsky, M.M. 1988, in Elemental Abundance Analyses, eds. S.J.

Adelman and T. Lanz (Inst. d'Astronomie, Univ. de Lausanne), p.179.

Elste, G.H. 1992, Ap.J., 384, 284.

Engleman, Jr., R. 1989, Ap. J., 340, 1170.

Faraggiana, R. and Gerbaldi, M. 1990, in Evolution in Astrophysics, ESA SP-310, p.39.

Faraggiana, R. Gerbaldi, M., van't Veer, C. and Floquet, M. 1988, Astr. Ap., 201, 259.

Fuhrmann, K. 1989, Astr. Ap. Suppl., 77, 345.

Gerbaldi, M., Floquet, M. and Hauck, B. 1985, Astr. Ap., 146, 341.

Gerbaldi, M., Floquet, M. Faraggiana, R. and van't Veer-Menneret, C. 1989, Astr. Ap. Suppl., 81, 127.

Gerbaldi, M. and Friere Ferrero, R. 1986, in New Insights in Astrophysics, ESA SP-263, p.311.

Gigas, D. 1986, Astr. Ap., 165, 170.

Glagolevskij, Yu, V., Panov, K. and Chuhakova, N.M. 1985, Pis'ma Astron. Zh., $11,749$.

Glagolevskij, Yu. V., Romanyuk, I.I., Najdenov, I.D., Shtol', V.G.

1989, Astrofiz. Issled. Izv. Spets. Astrofiz. Obs., $27,34$.

Glaspey, J.W., Michaud, G., Moffat, A.F.J. and Demers, S. 1989, Ap. J., 339, 926.

Griffin, R. and R. 1985, Astr. Ap., 149, 437.

Grevesse, N. 1991, in Evolution of Stars: The Photospheric Abundance Connection (IAU Sympos. No, 145), ed. G. Michaud and A. Tutukov, p.63.

Gulliver, A.F., Adelman, S. J., Cowley, C.R., Fletcher, J.M. 1991, Ap. J., 380, 223.

Gulyayev, S.A., Panchuk, V.E. and Pyatkes, S.G. 1987, Astrofiz.

Issled. Izv. Spets. Astrofiz. Obs. 25, 60.

Guthrie, B.N.G. 1985, MNRAS, 216, 1.

Guthrie, B.N.G. 1986, MNRAS, 220, 559.

Hannaford, P., Lowe, R.M., Grevesse, N., and Noels, A. 1992, Astr. Ap., 259, 391.

Hardorp, J., Cugier, H., Koratkar, A. and Scott, J. 1986, in New Insights in Astrophysics, ESA SP-263, p.377.

Hartoog, M.R. and Cowley, A.P. 1979, Ap.J. 228, 229

Hayes, D.S. 1985, in Calibration of Fundamental Stellar Quantities (IAU

Symp. No. 111, ed. D.S. Hayes, L.E. Pasinetti and A.G. Davis Phillip, p.225.

Hauck, B. and Slettebak, A. 1989, Astr. Ap., 214, 153.

Holweger, H., Bard, A., Kock, A. and Kock, M. 1991, Astr. Ap., $249,545$.

Holweger, H., Gigas, D. and Steffen, M. 1986, Astr. Ap., 155, 58.

Holweger, H., Steffen, M. and Gigas, D., 1986, Astr. Ap., 163, 333.

Isberg, B. and Litzén, U. 1985, Phys. Scripta, 31, 533.

Isberg, B. and Litzén, U. 1986, Phys. Scripta, 33, 420.

Jaschek, M. and Jaschek, C. 1987, Astr. Ap., 171, 380.

Klochkova, V.G. 1985, Pis'ma Astron. Zh., 11, 512.

Klochkova, V.G. and Kopylov, I.M. 1985, Astron. Zh., 62, 947. 
Knyazeva, L.N. and Kharitonov, A.V. 1990, Astron. Zh., 67, 1243.

Kopylov, I.M., Leushin, V.V., Sokolov, V.V., Topil'skaya, G.P., Tsymbal, V.V. and Gvozd', Yu. A. 1989a, Astrofiz. Issled. Izv. Spets. Astrofiz. Obs. 28, 59.

Kopylov, I.M., Leushin, V.V., Topil'skaya, G.P., Tsymbal, V.V., Gvozd', Yu. A. 1989b, Astrofiz. Issled. Izv. Spets. Astrofiz. Obs., 28, 72.

Lambert, D.L., McKinley, L.K. and Roby, S.W., 1986, PASP, 98, 927.

Lanz, T. and Artru, M-C. 1990, in Evolution in Astrophysics, ESA SP310 , p.275.

Lanz, T., Artru, M-C. and Didelon, P. 1990, in Evolution of Stars: The Photospheric Abundance Connection (IAU Sympos. No.145; Poster Papers), ed. G. Michaud, A. Tutukov and M. Bergevin (Univ. de Montréal), p.65.

Lebedev, V.S. 1990, Astrofiz. Issled. Izv. Spets. Astrofiz. Obs. $30,83$.

Leckrone, D.S. Wahlgren, G.M. and Johansson, S.G. 1991, Ap.J., 377, L37.

Leggatt, S.K. 1985, Astr.Ap., 153, 273.

Lemke, M. 1989, Astr. Ap., 225, 125.

Lemke, M. 1990, Astr. Ap., 240, 331.

Lester, J.B., Gray, R.O. and Kurucz, R.L. 1986, Ap. J. Suppl., 61, 509.

Leushin, V.V., Topil'skaya, G.P., Ryabschikova, T.A. and Pavlova, V.M. 1990, in Evolution of Stars: The Photospheric Abundance Connection (IAU Symposium No.145, Poster Papers), ed. G. Michaud, A. Tutukov and M. Bergevin (Univ. de Montréal), p.69.

Lopez-Garcia, Z. and Gargiulo, V. 1989, Ap. Lett. Comm., 27, 311.

Lyubimkov, L.S. 1989, Astrofizika, 30, 99.

Lyubimkov, L.S., Polosukhina, N.S. 1988, Iz. Krym. Astrofiz. Obs., 80, 30.

McNamara, B.J. 1987, Ap. J., 312, 778.

Malagnini, M.L. and Morossi, C. 1990, Astr. Ap., 85, 1015.

Mathys, G. and Cowley, C.R. 1992, Astr. Ap., 253, 199.

Mathys, G. and Lanz, T. 1990, Astr. Ap., 230, L21.

Moon, T.T. 1985, Commun. U. London Obs. No.78.

Moon, T.T. and Dworetsky, M.M. 1985, MNRAS, 217, 305.

Morossi, C. and Malagnini, M.L. 1985, Astr. Ap. Suppl., 60, 365.

Mountain, C.M., Leggatt, S. K., Selby, M.J., Blackwell, D.E. and Petford, A.D., 1985, Astr. Ap., 151, 399.

Napiwotzki, R., Schönberner, D. and Wenske, V. 1992, in The Atmospheres of Early-Type Stars (Lecture Notes in Physics, No. 401, Springer Verlag) ed. U. Heber, C.S. Jeffery, p. 18.

North, P. and Kroll, R. 1989, Astr. Ap. Suppl., 78, 325.

North P. and Nicolet, B. 1990, Astr. Ap., 228, 78.

Pan, X., Shao, M., Colavita, M.M., Armstrong, J.T., Mozurkewich, D., Vivekanand, M., Denison, C.S., Simon, R.S. and Johnston, K.J., 1992, Ap. J., 384, 624.

Pasinetti Fracassini, F., Castelli, F. and Fracassini, M. 1990, in Evolution of Stars: The Photospheric Abundance Connection (IAU Sympos. No.145; Poster Papers), ed. G. Michaud, A. Tutukov and M. Bergevin (Univ. de Montréal), p.57.

Ptitsyn, D.A. and Ryabchikova, T.A. 1986, Astron. Zh., 63, 527.

Ramella, M., Castelli, F., Malagnini, M.L., Morossi, C. and Pasian, F. 1987, Astr. Ap. Suppl., 69, 1. 
Ramella, M., Gerbaldi, M., Faraggiana, R., and Böhm, C. 1989, Astr. Ap., 209, 233.

Relyea, L.J. and Kuruez, R.L. 1978, Ap. J. Suppl., 37, 45.

Roby, S.W. and Lambert, D.L. 1990, Ap. J . Suppl., 73, 67.

Roby, S.W., Leckrone, D.S. and Adelman, S.J. 1989, Bull. AAS, 21, 1198.

Ruban, E.V. 1988, Astron. Tsirk. No.1532, p.29.

Ryabchikova, T.A. and Smirnov, Yu. M. 1988. Astron. Tsirk. No.1534, p.21.

Ryabchikova, T.A. and Piskunov, N.E. 1988, in Magnetic Stars, ed. Yu. V. Glagolevsky and I.M. Kopylov (Leningrad), p.124.

Sadakane, K. 1991, PASP, 103, 355.

Sadakane, K., Jugaku, J., Takada-Hidai, M. 1985, Ap. J ., 297, 240.

Sadakane, K., Jugaku, J. and Takada-Hidai, M. 1988a, Ap. J., 325, 776.

Sadakane, K., Jugaku, J. and Takada-Hidai, M. 1988b, PASP, 100, 811.

Sadakane, K., Nishimura, M. and Hirata, R. 1986, Publs. Astr. Soc. Jpn., 38, 215.

Sadakane, K., and Ueta, M. 1989, Publs. Astr. Soc. Jpn., 41, 279.

Savanov, I.S. 1985a, Izv. Krym. Astrofiz. Obs., 71, 144.

Savanov, I.S. 1985b, Izv. Krym. Astrofiz. Obs. 73, 92.

Savanov, I.S. 1987, Izv. Krym. Astrofiz. Obs., 76, 37.

Savanov, I.S. and Khalilov, A.M. 1986, Izv. Krym. Astrofiz. Obs. 72, 106.

Schneider, H. 1987, Hvar Obs. Bull., 11, 29.

Smith, K.C. 1992, Ph.D. Thesis, University of London.

Smith, K.C. and Dworetsky, M.M. 1990a, in Evolution in Astrophysics, ESA SP-310, p.279.

Smith, K.C. and Dworetsky, M.M. 1990b, in Evolution of Stars: The

Photospheric Abundance Connection (IAU Sympos. No.145,

Poster Papers), ed. G. Michaud, A. Tutukov, M. Bergevin (Univ. de Montréal), p.81.

Stenflo, J.O. and Lindegren, L. 1977, Astr. Ap., 59, 367.

Stürenburg, S., and Holweger, H. 1990, Astr. Ap., 237, 125.

Stürenburg, S., and Holweger, H. (Erratum) 1991, $246,644$.

Takada-Hidai, M. 1990, in Evolution of Stars: The Photospheric

Abundance Connection (IAU Sympos. No.145, Poster Papers), ed.

G. Michaud, A. Tutukov and M. Bergevin (Univ. de Montréal), p.85.

Takada-Hidai, M. 1991, in Evolution of Stars: The Photospheric

Abundance Connection, (IAU Sympos. No.145) ed. G. Michaud and A. Tutukov, p.137.

Takada-Hidai, M. and Jugaku, J. 1992, PASP, 104, 106.

Takada-Hidai, M., Sadakane, K. and Jugaku, J. 1986, Ap.J., 304, 425.

van Santvoort, J. 1987, in Astrochemistry (IAU Sympos. No.120), ed.

M.S. Vardya and S.P. Tarafdar, p.392

Van Winckel, H., Mathis, J.S., Waelkens, C. 1992, Nature, 356, 500.

Venn, K.A. and Lambert, D. L. 1990, Ap. J., 363, 234.

Wahlgren, G.M., Leckrone, D.S., Johansson, Se. and Rosberg, M. 1992, Bull. AAS, 24, 767.

Zöchling, J. and Muthsam, H. 1987, Astr. Ap., 176, 75.

Zverko, J., Ziznovsky, J., Zboril, M., Hric, L., Polosukhina, N., Malanushenko, V., Bychkov, V., Iliev, I. 1987, Proc. 1Oth European Regional Meeting of the IAU (Publs. Astr. Inst. Czech. Acad. Sci. No. 70), p.71. 